\title{
¿HEGEL FILÓSOFO DE LA DIFERENCIA? REFLEXIONES SOBRE LA CONCEPCIÓN HEGELIANA DE LA IDENTIDAD
}

\author{
Por: Camilo Andrés Morales* \\ camilo.morales@udea.edu.co
}

Existe un talento para separar lo que nunca está separado y aislar en el pensamiento lo que en la naturaleza está siempre bien unido. Este [talento] es imprescindible para filosofar, pero se traduce en un talento desgraciado y especulativo, a menos que aparezca vinculado con el talento filosófico para reunir lo que se ha separado, pues sólo ambos talentos unidos hacen al filósofo (Schelling, 2006: 53)

RESUMEN La filosofía hegeliana en general, y en particular la "Ciencia de la lógica" y el tratamiento que en esta se hace sobre nociones como las de identidad y diferencia, generaron desde el momento mismo en que vio la luz, un sinnúmero de posiciones críticas tales como las de Schelling, los hegelianos de izquierda y, en general, de todos aquellos filósofos que, en virtud de las posibles implicaciones prácticas de una filosofía de la identidad buscaron "expurgar la semilla del dragón del panteísmo hegeliano", entre las cuales es necesario mencionar la legitimación de estados totalitarios y los genocidios asociados a ellos. Esta búsqueda, fue realizada sobre la afirmación de que el pensamiento hegeliano, al estar soportado sobre la identidad (absoluto, estado, etc.), implica una negación y, por tanto, aniquilación y reducción de la diferencia. En este sentido, el presente artículo busca examinar la noción hegeliana de la identidad, la correspondencia o no de las críticas que sobre el particular

1 Este artículo es resultado del proyecto de investigación sobre Antropología política en Kant y Hegel, el cual fue financiado por el Comité para el Desarrollo de la Investigación -CODI- de la Universidad de Antioquia. De otro lado, agradezco las observaciones hechas por el evaluador del texto, las cuales sin duda contribuyeron para lograr de mejor forma los objetivos del manuscrito original.

* Instituto de Filosofia - Universidad de Antioquia - Grupo de Investigación en Filosofia Politica. Artigo recebido em 01/11/16 e aprovado em 01/02/17 
se han hecho sobre Hegel y, finalmente, analizar una de las salidas que, desde las interpretaciones hegelianas más recientes, se han hecho para responder a las críticas: la filosofía hegeliana in stricto sensu, una filosofía de la diferencia.

Palabras clave: identidad, diferencia, razón, entendimiento, absoluto.

Abstract: The Hegelian philosophy in general, and in particular the "Science of Logic" and the treatment that in this is made about notions as identity and difference, generated since its conception a countless critical positions such as those of Schelling, the left Hegelian and, in general, of all those philosophers who, accordingly with the posible practical implications of a philosophy of identity sought to "purge the dragon's seed from Hegelian pantheism" among which it is worth to mention the legitimization of totalitarian states and genocide associated with them. This search was made on the assertion that Hegelian thought, being supported on the identity (absolute, State, etc.), implies a negation and, therefore, annihilation and reduction of the difference. In this sense, the present article seeks to examine the Hegelian notion of identity, the correspondence or not of the criticisms that have been made about Hegel and, finally, to analyze one of the outlets that, from the most recent Hegelian interpretations, have been made to respond to the criticisms: the Hegelian philosophy in stricto sensu, a philosophy of the difference.

Keywords: identity, difference, reason, understanding, absolute.

Por todos son conocidas las críticas que se le han hecho a Hegel casi desde el mismo momento en el cual es publicado su sistema (Bauer, Meineker, Schelling, Schopenhauer et. al.). Para ilustrar esto, basta recordar sólo el hecho de que no más de 11 años después de la publicación de la última edición de la Ciencia de la lógica, en 1841, Schelling fue llamado a Berlín por el Rey de Prusia para expurgar "la semilla del dragón del panteísmo hegeliano" y su "superficial omnisciencia" (Toews, 1980. p. 254). Sin embargo, esto no significa que dichas críticas sólo hayan tenido lugar en el horizonte inmediato de Hegel. Antes bien, ellas han trascendido en el tiempo y han tomado distintos matices, pero se han concentrado, sobre todo, en temas como la supuesta defensa y legitimación hegeliana del estado prusiano, su negación del principio de no-contradicción y, como consecuencia de esta, su afirmación y defensa a ultranza de la identidad por encima de la diferencia (el recordado totalitarismo como sinónimo de sistematismo que desde los primeros "posthegelianos" se le endilgara a Hegel). 
Pero, todo ello, y las respuestas que desde Hegel puedan darse, depende de qué tipo de hegeliano o de crítico de Hegel sea, como bien los distinguiera Paul Ashton: 1. aquellos que toman una aproximación temática a la filosofía de Hegel, principalmente desde su filosofía política, evitando las implicaciones de la lógica especulativa; 2 . aquellos que intentan trabajar a través de los textos sistemáticos sin desconocer las necesarias relaciones con la filosofía práctica (Ashton, 2008. p. 315).

En el presente trabajo se tomará partido por la segunda vertiente que define Ashton para enfrentarse a Hegel, pues creo que, como lo dice el autor, es en el ámbito de la Ciencia de la lógica donde se tematiza la estructura del pensamiento o, dicho con Hegel, donde el pensamiento se piensa a sí mismo, lo cual antes de ser una afirmación teñida de la más pura y simple metafísica, obedece, por un lado, a la intención que, desde Kant (de tal suerte que el kantismo de Hegel, como se ha llamado, no sólo se reduce a la Vida de Jesús) (para referirme al contexto inmediato de Hegel), ha caracterizado a la filosofía, esto es: ser crítica y, por otro, a que es en la Ciencia de la lógica donde se estructura conceptualmente la forma como pensamos y como es la realidad (de allí el idealismo absoluto en el cual se ubica la filosofía hegeliana). Sin embargo, esto no significa que la otra alternativa de lectura sea deleznable per se, sólo que pienso que es en la Ciencia de la lógica donde se hayan los elementos conceptuales para tratar el problema que aquí me convoca, a saber, el de la identidad.

Como se afirmó líneas atrás, muchas y de muchos tipos han sido las críticas que se han emitido contra Hegel, a las cuales no me referiré por su extensión y porque no es mi intención hacer una defensa cuasi ferviente del pensamiento de Hegel. Lo que sí pretendo es revisar el problema de la identidad en Hegel, para desde allí referirme críticamente a las afirmaciones según las cuales Hegel es el filósofo consumado de la identidad y, por tal motivo, negador de la diferencia. Sin embargo, mi reflexión intenta dar un paso más, no necesariamente en el sentido de ser vanguardista, sino con el fin de entablar un diálogo con los estudios más actuales sobre la filosofía hegeliana, que son vastos y sobre temas y relaciones aún en fermentación, como aquellos que vinculan a Hegel con la filosofía analítica, el pragmatismo, etc. ${ }^{1}$ Dicho diálogo tendrá como interlocutores las posiciones de algunos filósofos contemporáneos, encarnados ellos en William Maker y quien fuera vicepresidente de la Sociedad Americana de Hegel y uno de los intérpretes actuales del pensamiento hegeliano. La tesis de Maker, que motiva además el título de esta presentación, es que "Hegel es

1 Además de las conocidas lecturas que de Hegel hicieron McDowell (1996) y Brandom (2014), análisis como los de Tom Rockmore (2005), en especial los capítulos 2 y 3, así como los de Redding (2007) y Nuzzo (2010), se han convertido, con el paso del tiempo, en referencias clásicas y obligadas para la revisión contemporánea de la filosofía hegeliana $y$, por tanto, para algunas de las preguntas siempre presentes sobre los filósofos clásicos: ¿por qué y para qué leerlos hoy? 
el filósofo de la diferencia", de la cual comparto sus motivaciones, pero no su desenlace que, valga la pena anticiparlo, juzgo tan desafortunado como aquel que afirma lo contrario, a saber, que Hegel es el filósofo de la identidad.

El suelo argumentativo de mi reflexión, como dije antes, será la Ciencia de la lógica, pero es necesario no perder de vista el anclaje práctico que tanto esta obra en particular, como el pensamiento de Hegel en general tienen. Desconocerlo, sería pensar que el sistema del filósofo alemán será simple metafísica o filosofía edificante o esotérica ${ }^{3}$. Para llevar a buen este trabajó es procedente seguir los siguientes pasos: en primer lugar es necesario situar a Hegel en la Alemania de su tiempo, pues si como él mismo advirtiera en varios de sus escritos $^{4}$ la filosofía es hija de su tiempo, únicamente comprendiendo algunas características del suyo podrá entender no sólo el pensamiento de Hegel, sino también comprender por qué su proyecto no sólo es viable, sino necesario en dicho tiempo, lo cual no es un simple embeleco suyo sino el proyecto de buena parte de los intelectuales de su tiempo (Lessing, Herder, Goethe, Schiller, etc.), convirtiendo la pregunta por la identidad en un tema casi de interés nacional. En segundo lugar serán mecionadas algunas de las críticas que se le han hecho a Hegel y a su concepto de Identidad. En tercer lugar, será expuesta brevemente la respuesta de Maker a dichas críticas y, acto seguido, tendrá lugar la exposición la estructura de la identidad desde la Ciencia de la lógica para, en último lugar, ofrecer algunas conclusiones.

\section{Hegel. Hacia la luz del sol.}

Esta expresión ("hacia la luz del sol"), es el subtítulo del extraordinario libro de H.S. Harris (1972) dedicado no sólo a hacer un recorrido por la vida del joven Hegel, como denominara Nohl al periodo previo a la publicación del Escrito sobre la diferencia y a la Fenomenología del espíritu, sino a exponer la forma como se configuró el pensamiento hegeliano y las relaciones que el autor tuvo con su tiempo, cultura y configuración socio-política del mismo. Pero, ¿desde dónde se va hacia la luz del sol? ¿por qué es necesario tal tránsito? Y, fundamentalmente, ¿qué significa la luz del sol? Baste por el momento decir, a propósito de la última pregunta y para comprender el sentido del subtítulo, que la luz del sol remite sin ninguna duda a la alegoría de la caverna expuesta por Platón en el libro VII de la República, pero también, y fundamentalmente, a la luz propia de la Aufklärung, es decir, a la luz de la razón, como explica Harris al comienzo de su libro. 
Esta idea, y la metáfora que la contiene, dan cuenta de que hay un momento previo al brillo de la razón (Vernunft) y uno que constituye la luz de la razón, pero baste decir, no a uno después de la luz del sol, para dejar de lado aquellas lecturas que afirman que Hegel predijo algo o que afirmó que evolucionábamos hacia algo (Vide Avineri (1972)), justificando con ello un supuesto nacionalismo al estilo del nacional socialismo, el estado prusiano o cualquiera de los regímenes totalitarios. Para decirlo desde ya, ese momento previo a la luz de la razón no es más que la incertidumbre política y religiosa que caracterizó por un buen tiempo a Alemania ${ }^{5}$.

En este sentido, basta darle un paso a la historia de dicho país, incluso uno ligero y desprevenido, para notar que ha sido una historia de reformas, convulsiones y guerras, pues los conflictos religiosos y políticos han estado a la orden del día en el país teutón. Desde el siglo XV, piensen por ejemplo en la dinastía de los Luxemburgos, e incluso antes, pasando por el joven Maximiliano hasta la reforma luterana, la Guerra de los treinta años y la paz de Westfalia, junto con la distribución espacial de Alemania producto de la confederación del Rihn, la "cultura de la violencia" ha sido un factor constitutivo del país del norte. Evidencias históricas muestran que dicha violencia sumió a Alemania, en distintos momentos, en un "estado de caos" donde, según los testimonios del comerciante Jacob Wagner y del monje Reginbald Moehner, hallados en sus diarios, "el hambre se agrava[ba] día a día, hora a hora, de tal suerte que la gente remoja[ba], corta[ba] y [cocinaba] las pieles de los bueyes, las vacas y otros animales...[y] los soldados golpeaban y les disparaban a perros y gatos [con el fin de comérselos]. Ahora casi ningún animal se ve en la ciudad" (Roeck, 2006. pp. 232-233). Dicho panorama se produjo a la par con un estado de caos y destrucción, donde la restauración del orden y la armonía era un imperativo, afirmaciones como la de un cardenal romano según el cual "toda Alemania [era] una pandilla de bandidos" (Brady, 2009. p. 23).

Lo anterior no puede ser un detalle más, o un mero adorno textual, pues al interior del pensamiento hegeliano, como es sabido y señalado constantemente, se exige que la filosofía tenga un origen histórico determinado, esto es, que sea "hija de su tiempo" (Hegel, 2000. §§ 6-13). Esta exigencia, que Hegel comparte, por ejemplo, con uno de los más destacados filósofos y dramaturgos del Sturm und Drang, Friedrich Schiller (Schiller, 1975. pp. 69-83), es garante,

5 Imagen y diagnóstico de ello es lo expuesto, entre otros, por Heinrich von Kleist (2010) en "Michael Kohlhaas", donde Alemania se presenta en llamas ante la imposibilidad de materialización de un principio moral y legal que permita, para el caso de Kohlhaas, la reparación por el agravio moral sufrido. A lo cual habría que añadir lo expresado por Friedrich Schiller (1975) en la quinta de sus cartas sobre la educación estética del hombre: "El hombre se refleja en sus hechos, y ¡qué espectáculo nos ofrece el drama de nuestro tiempo! Por un lado, salvajismo, por el otro apatía: ¡los dos casos extremos de la decadencia humana, y ambos presentes en una misma época!". (Schiller, 1975. p. 65). 
por un lado, del anclaje práctico que debe tener el pensamiento filosófico y, por otro lado, es al mismo tiempo un llamado a que la filosofía, en tanto consideración pensante del tiempo histórico en el cual surge, sea crítica, reflexiva y por qué no, transformadora del mismo.

Según esto, y de consuno con el tema de esta presentación, el problema de la identidad no está por fuera de este anclaje práctico. Antes bien, es la identidad uno de los conceptos que está más inmerso en el devenir histórico-social de, para nuestro caso, la Alemania del tiempo de y anterior a Hegel, debido a que ella emerge, como exigencia y alternativa, a siglos de caos, desunión y desmembramiento de la sociedad alemana. Este paisaje, entonces, es el contexto en el cual surge y se forma una figura como la de Hegel, por lo cual es menester describirlo un poco más en detalle (para lo que interesa en este momento, claro está: señalar porqué la identidad tiene raíces histórico-sociales profundas y, por tanto, por qué hablar de "problema" de la identidad).

Como señala Robert C. Solomon (1983, p. 33), “[e]1 mundo de Hegel fue un estado de caos", el cual se remonta hasta el tiempo de La Reforma. Esta, como es sabido, surge como producto de las acciones de Martin Lutero dirigidas contra Roma, contra la Iglesia, las cuales estuvieron motivadas por "un resentimiento nacional contra el papado, el cual, por su cooperación con los grandes feudos en contra del Emperador, impidió la unidad nacional" (Taylor, 2001. p. 9). Este apoyo de Roma a los grandes feudos convirtió a la religión en un espacio de corrupción y decadencia, donde los obispos, por ejemplo, eran nombrados por el Rey, y no por, digamos, su bondad, conmiseración o vocación de pastores, lo cual condujo, entre otras cosas, a que los pecados y el mal fueran, en palabras de Hegel, "expiados con dinero" (Hegel, 1994. p. 657), situación que a la postre implicó la pérdida de legitimidad y autoridad de la Iglesia. Pese a este agreste panorama, en principio, la Reforma impulsada por Lutero tuvo un tinte pacífico, pero conforme avanzó hacia la puesta en cuestión de los dogmas fundamentales de la Iglesia, ello implicó una separación necesaria entre religiones, lo cual condujo, indefectiblemente, a la violencia aterradora de la Guerra de los treinta años, donde Alemania quedó, además de devastada y desolada, dividida, pues, a pesar del tratado de la Paz de Westfalia, la unificación religiosa y política fue imposible, ya que al reconocérsele independencia y legitimidad a la Iglesia protestante ello se convirtió en una terrible humillación para la Católica.

En este orden de ideas, frente al anquilosamiento y la degradación del cristianismo, la Reforma se planteó como alternativa para elevar la libertad individual sobre el universalismo cristiano, resultando esto, según algunos intérpretes, en un particularismo radical que imposibilitaría para siempre la unidad. Esta fragmentación alemana tuvo grandes consecuencias negativas para 
la reconstrucción de un estado de unidad, pues "[d]esgraciadamente, los príncipes intervinieron en esta desunión, y muchos de ellos usaron estas disputas para fortalecer y extender sus ganancias (económicas) y su poder como gobernadores provinciales" (Novalis, 1997. p. 141). Prueba de ello es, por ejemplo, la división de Alemania en lo que se ha llamado "234 insignificantes estados" (Vide Solomon, 1983. p. 36) separados entre sí, que fue el horizonte que tenía Hegel ante sus ojos en 1806, año en el cual Hegel escribiría la mayor parte de su Fenomenología del espíritu. Esta gran diseminación del pueblo alemán y sus constantes convulsiones provocaron que la identidad, nacional en varios sentidos, surgiera en el horizonte, más que para tematizarla, para construir una, esto es, como algo que se reclama en virtud de la absoluta y descarnadora diversidad. Esto tiene sentido en la medida en que se piensa, con y a partir de Hegel, que la "identidad atomística, individual, no es identidad" (Solomon, 1983. p. 41), pues para construir algo llamado identidad, en sentido hegeliano, es menester dar cuenta, no sólo externa, sino también internamente, de la diferencia como parte constitutiva y fundamental de la identidad, pero esto lo veremos más adelante.

Como se ve, la Alemania en la cual habitaba Hegel era todo menos un país en calma y dedicado apaciblemente al pensamiento. Pero, ¿qué vinculo guarda este pequeño panorama con la filosofía de Hegel y, más aún, con el asunto de la identidad? En primer lugar, y en sentido general, al ser la filosofía hija de su tiempo, el panorama mostrado a grosso modo sirve como escenario sobre el cual surge la filosofía hegeliana y como el contexto al cual van dirigidas sus respuestas, propuestas, críticas, etc. Por otro lado, y hablando desde dentro de la filosofía misma de Hegel, este breve panorama es útil en la medida en que "sirve" para comprender un poco más dos asuntos de vital importancia para esta presentación y relacionados entre sí: la necesidad de la filosofía (Bedürfnis der Philosophie) y el problema de la identidad propiamente dicho. Con respecto a lo primero, Hegel afirma, en uno de sus primeros escritos filosóficos, que "la necesidad de la filosofía surge cuando el poder de unificación desaparece de la vida de los hombres, y los opuestos pierden su viva relación e interacción y cobran autonomía" (Hegel, 1990. p. 20), de donde se colige que la filosofía en general, y la suya en particular, emerge no como un capricho de un hombre, o como producto de un trance místico-religioso, sino como respuesta a un tiempo determinado por convulsiones políticas y religiosas particulares, apenas mencionadas con antelación. Así, podríamos decir en este momento que la filosofía hegeliana es necesaria, para su tiempo, en la medida en que se erige como alternativa para reconciliar las oposiciones que, como se vio, le hicieron tanto mal a Alemania e imposibilitaron la creación de una identidad nacional.

Esta creación de la Identidad nacional será abordada desde diferentes perspectivas por los múltiples filósofos y literatos alemanes y no por los po- 
líticos o militares, pues tras la salida de Napoleón del panorama alemán, la influencia político-militar del pueblo teutón fue casi nula (Solomon, 1983. p. 37 y ss.). Por ejemplo, un proyecto importante de construcción de la identidad nacional estuvo dado a partir del romanticismo alemán y, al interior de este, por una figura como Novalis. Este, sumergido en el estado caótico mencionado con antelación y antes que superado, exacerbado por el protestantismo, opta por la cristiandad, por el cristianismo, como estrategia para llevar a Alemania de nuevo a la paz y a la cohesión. Así, dice Novalis que:

La cristiandad debe transformarse de nuevo en algo vivo y eficaz, y formar de nuevo una Iglesia visible sin admiración por las fronteras nacionales, la cual tomará sobre su pecho las almas sedientes de lo sobrenatural, y gustosamente se convertirá en mediadora entre el antiguo y el nuevo mundo (Novalis, 1997. p. 151).

No obstante, la propuesta hegeliana no se inscribe en el contexto del romanticismo y, para el caso de Novalis, en la pretensión de re-construir la cristiandad o producir una nueva mitología. Antes bien, Hegel, como hijo de su tiempo, es decir, como moderno, piensa en la reconciliación de los opuestos desde la perspectiva de la razón (Vernunft), que es "el punto de convergencia de conflictos y el espacio de su inevitable resolución" (Nuzzo, 2008. p. 21) y, por tanto, desde un ambiente filosófico y no poético o literario. Por tal motivo, ante la pregunta por la necesidad de la filosofía y, en consonancia con ello, frente al problema de la identidad como proyecto que reconcilie a Alemania consigo misma, pero también al hombre consigo y con el estado y con la religión, la filosofía hegeliana se plantea como un esfuerzo no sólo por pensar su tiempo, sus necesidades y limitaciones, sino también por transformarlo teniendo dicha transformación un sustento en la razón. Por tal motivo, es momento de centrarse en la filosofía hegeliana y su construcción de la identidad, eso sí, sin perder de vista su pertenencia histórica.

\section{Críticas a la noción hegeliana de la identidad: la respuesta de Maker.}

Casi desde el momento mismo en el que Hegel publica su sistema, surgen posiciones críticas con respecto a varios de los conceptos más relevantes de su filosofía, tales como el de Absoluto, Idea absoluta, Eticidad, Identidad, Diferencia, Fundamento, entre otros. Dichas críticas comienzan con Schelling, Meineke, Fries y Feuerbach, y pasan por Marx (el joven Marx), Adorno y Marcuse, hasta llegar a Deleuze, Derrida (Positions) y Lèvinas, y más recientemente, a filósofos como William Desmond (1992), por mencionar sólo algunos. Tales críticas, dirigidas unas al estilo oscuro y denso de la escritura de Hegel, y otras a asuntos más de fondo como el de lo absoluto, la totalidad y la identidad, 
han propiciado lo que se ha llamado "los mitos y leyendas de Hegel", como bien los denominara Jon Stewart (1996). Como no es de interés aquí evaluar cada uno de esos supuestos mitos y leyendas, el énfasis será puesto en resumir lo más sucinta y claramente posible las críticas dirigidas a atacar la filosofía hegeliana como una filosofía consumada de la identidad.

Un buen comienzo para esta tarea pasa por recordar las palabras de Adorno (2008), donde el cofundador de la Escuela de Frankfurt afirma que si bien el gran logro de la filosofía hegeliana es la inclusión de la contradicción en el ámbito del pensamiento, relacionado este con el mundo (por ejemplo hay contradicción entre sujeto y objeto, entre hombre y sociedad, etc.), dicho logro se ve opacado, y si se quiere refutado, por el hecho de que la dialéctica hegeliana termine en una unidad que contiene los momentos anteriores, lo cual, según Adorno, anularía la contradicción misma pues el absoluto, según el frankfurtiano, sería una instancia superior y omniabarcante que se erigiría como omnipotente frente a las particularidades de lo diferente. En este sentido, la afirmación hecha por Hegel al comienzo de la Ciencia de la lógica según la cual la Idea es una "identidad de identidad y diferencia" pondría un énfasis superlativo en la identidad, subsumiendo la diferencia en esta, lo cual, contrario a ser un problema de jerga filosófica, o de fundamentación metafísica de un sistema filosófico, tendría implicaciones nefastas, ya que a partir de dicha argumentación se podría justificar, según Adorno, la instauración de sistemas totalitarios y, por ende, la creación de campos de concentración, en los cuales, para introducir otro de los autores que elijo para ejemplificar las críticas a la filosofía hegeliana como es Deleuze, "la diferencia es crucificada...[pues ella] únicamente deviene un objeto de representación sólo en relación a una identidad concebida, una analogía juzgada, una oposición imaginada o una similitud percibida" (Deleuze, 1993. p. 180).

El problema de esta aparente reducción de la diferencia a la identidad es, al menos, doble, pues por un lado tiene nefastas implicaciones políticas, como ya se mencionó, y que se evidencian, por ejemplo, en Auschwitz, donde según Adorno no morían individuos sino ejemplares, es decir, la existencia de tales individuos era indiferente o incluso peligrosa para el mantenimiento del statu quo (Adorno, 2008. p. 332). Por tal motivo, había dos alternativas: 1. ignorar la diferencia o, 2. como diría Deleuze, crucificarla, lo que tal parece fue, y valdría la pena incluso revisar este tiempo verbal, el caso en los sistemas totalitarios. Pero, por otro lado, dicha aniquilación de la diferencia tiene graves implicaciones para la filosofía misma, pues ella implicaría la posibilidad de instaurar verdades eternas, atemporales, a-espaciales y, por tanto, abstractas, restándole vitalidad y realidad al discurso filosófico, que es lo único que dejaría abierta la posibilidad para la filosofía después de Auschwitz. 
A este tipo de críticas le han comenzado a salir al paso, y digo que le han comenzado a salir al paso porque sobre todo en el mundo anglosajón hay una especie de revival de la filosofía hegeliana, algunas reflexiones de autores que, como William Maker, piensan que nada hay más alejado de la realidad que la afirmación según la cual Hegel hace abstracción de la diferencia y que, por tanto, su tratamiento de ésta no es más que un momento pasajero en el desarrollo de la Ciencia de la lógica hacia la instauración de una entidad absoluta.

Es así como William Maker (2007), con el objetivo de refutar la idea según la cual la filosofía hegeliana es acusada de absolutismo reduccionista, afirma que "Hegel es el filósofo de la diferencia, de la otredad y la no identidad" (Maker, 2007. p. 16). Esto lo hace haciendo énfasis en dos momentos de la Ciencia de la lógica, a saber: la primera aparición del concepto de devenir (Werden) en la Doctrina del ser y el comienzo de la tercera parte del mismo texto, la cual está dedicada al concepto (Begriff). En ambos momentos, tanto al inicio como al final de la Lógica, según Maker, se muestra como necesario y no como algo posible o contingente el hecho de que, por ejemplo, el ser y la nada sólo sean lo que son en la medida en que "uno traspase al otro", lo cual implica que cada categoría del pensamiento tiene no sólo su razón de ser sino su posibilidad en virtud de la existencia y la relación con su contraria. Es así como a lo largo de la Lógica aparecen duplos de conceptos, tales como ser/ nada, esencia/apariencia, concepto/objeto, etc., recordando con ello el epígrafe de Schelling el cual caracteriza, a su vez, a la dinámica del pensamiento filosófico en el idealismo absoluto alemán (Schelling y Hegel) como constituido por el entendimiento (Verstand). ${ }^{6}$

Por otro lado, y con supuesta mayor claridad, lo anterior se evidencia en el paso de la Doctrina del ser a la doctrina de la esencia, donde, en primera instancia, y sólo en primera instancia, parece que lo esencial es la esencia, es decir, el ámbito de la reflexión y sus categorías, mientras que lo que es, simplemente es inesencial. No obstante, como bien Hegel lo señala, lo que en un primer momento se toma como inesencial, luego del uso de la razón se concluirá que es esencial, pues al ser un momento del despliegue, por ejemplo, del ser a la idea o de la certeza sensible al Saber absoluto, este constituye, según Maker, la condición de posibilidad del momento siguiente. Esto, que en principio parecería un mero discurso solipsista, bien podría ejemplificarse en la Filosofía del Derecho con la "díada" moralidad y eticidad, donde si bien a primera vista la eticidad es lo esencial, pues es el momento de la realización plena de la libertad, no por ello la moralidad es inesencial -y menos aún el derecho abstracto-, 
pues en virtud de la noción hegeliana de superación (Aufhebung) todo momento anterior estará contenido en el próximo.

Lo anterior le sirve a Maker para dejar, según él, dos cosas claras: 1. que en la filosofía hegeliana "la identidad es imposible sin la articulación de lo irreductible, de la diferencia" y, en este sentido, la diferencia y lo no-idéntico, no son meras variaciones de la identidad, sino un factor constitutivo e imprescindible en la comprensión de la misma identidad (Ibíd., p. 19); y 2. que "el gran problema de los críticos es que ellos ignoran el método de Hegel[...] y su intento por articular, describir y glorificar la otredad, la diferencia" (Ibíd., p. 27).

Más allá de Maker. La concepción hegeliana de la identidad a la luz del principio de la doble transición.

Si bien mi posición es cercana a la de Maker debido a que también yo pienso que en Hegel no se da una abstracción de la diferencia, pues ésta es parte constitutiva y fundamental de la identidad, de manera que podría decirse lo mismo de manera inversa, pienso que la solución del mencionado autor adolece de lo mismo que él critica. Para sustentar esto, echaré mano junto con Robert Williams de la tesis de la doble transición (gedoppelte Übergang), la cual Hegel introducirá en la segunda edición de la Ciencia de la lógica de 1832 (Hegel, 1969. p. 384.), la cual sugiere que Hegel mismo ha sido consciente de un posible malentendido que puede generarse si se lee la Ciencia de la lógica desde la óptica del entendimiento y del lenguaje ordinario.

Esta tesis de la doble transición afirma que, para poder hablar de totalidad (¡no de totalitarismo!), es necesaria una doble transición, la cual, además de implicar que para hablar de identidad es necesario pensar en la diferencia, también lo contrario es válido, es decir, que para poder hablar de diferencia es necesaria la identidad.

Pero, miremos un poco más de cerca qué significa esto a la luz del desarrollo del pensamiento hegeliano en general y de la Ciencia de la lógica en particular. Desde los comienzos filosóficos de Hegel, el problema de la identidad tuvo un lugar preponderante en su itinerario filosófico. Así, un escrito de 1801, llamado Diferencia entre los sistemas de filosofía de Fichte y Schelling, se constituye en una de las primeras fuentes para rastrear la noción de identidad en el pensamiento del filósofo alemán, o al menos la más conocida, pues desde el Fragmento de Tubinga es posible ya hacer dicho rastreo. En dicho texto, ante la posibilidad de adoptar, en filosofía, un principio en la forma de una proposición que determine toda forma de conocimiento, de manera que cumpla la función de premisa y fuente de todo lo que del mundo y de nosotros mismos conocemos, Hegel asegura que la absolutez de dicho principio, o su completud, no es tal. No en el sentido de que pueda ser absoluta para unos y para otros no, sino en el sentido de que dicha proposición, expresada 
como $\mathrm{A}=\mathrm{A}$, es relativa al obrar propio del entendimiento (Verstand) que, como lo declara en la Ciencia de la lógica, abstrae, "determina y mantiene firmes las determinaciones", por lo cual la posibilidad de un conocimiento real, donde los opuestos estén reconciliados (Wirklichkeit), sucumbe ante el formalismo propio de lo que Adorno llamara pensamiento identificador, pues lo que se suprime con dicha identidad es el contenido de A, esto es, sus determinaciones. Por tal motivo, la identidad, expresada por el entendimiento de manera formal, es vista por Hegel como no absoluta y, por tanto, como una enunciación abstracta y preliminar de dicho concepto, esto es, una "pura igualdad" (Jaeschke, 2003. p. 113).

Esta consecuencia la extrae Hegel del análisis de la proposición $\mathrm{A}=\mathrm{A}$, pero atañe también a la proposición $\mathrm{Yo}=\mathrm{Yo}$, cuyo referente más inmediato para la filosofía hegeliana es el pensamiento de Fichte. Dicho análisis, que busca indagar por la absolutez de tal principio, lo emprende Hegel desde la estructura interna del mismo, lo cual lo lleva, en primer lugar, a plantearse la pregunta por lo que no es A, esto es, B. La existencia de este no ser A queda, en la formulación de dicho principio, abstraída. Así, en la proposición mencionada no aparece B, sino que lo absoluto se concibe de forma exclusivamente autorreferencial, es decir, se instaura a sí mismo como verdad a partir de un ejercicio abstrayente de la diferencia. Por tal motivo, el principio de identidad, formulado en estos términos es, para Hegel, tan absoluto como el principio de no contradicción o de evitar la contradicción, es decir, carece de absolutez pues está condicionado por la abstracción de la diferencia. Este tipo de identidad, por tanto, gracias a ser un producto del entendimiento, es insuficiente ante las pretensiones de la filosofía hegeliana, pues esta tiene como finalidad (Zweck) la instauración de la reconciliación entre los opuestos o, para nuestro caso, la reconciliación entre la identidad y la diferencia, lo que a la postre implicará, como será mostrado más adelante, la formulación y/o consolidación de una identidad en estrecha relación con la diferencia.

En primer lugar es necesario advertir, como deriva ineludible de lo señalado hasta este momento, que la identidad no es posible enunciarla en términos de una proposición o enunciado, pues su mera enunciación implicaría, ipso facto, la supresión o abstracción de la diferencia. Así, una tematización lógica de la identidad, en sentido especulativo, que supere dicha concepción formal, la lleva a cabo Hegel en la Ciencia de la lógica en general y, en particular, en la Doctrina de la esencia. Es aquí donde introduce Hegel su investigación sobre la identidad, la cual, como se verá, tiene varios momentos.

Sin embargo, en el contexto de este numeral, baste decir que esta primera forma de la identidad, entendida como autorreferencialidad, Hegel la denomina formal o identidad del entendimiento (Hegel, 2000. § 115), pues aquí podría enunciársele del siguiente modo: "el ser es idéntico a sí mismo" o, lo que es 
lo mismo, $\mathrm{A}=\mathrm{A}$. En este primer modo de la identidad, como ya se mencionó, las determinaciones, por ejemplo, del Ser, aparecen como algo distinto, como algo que está lejos de la esencia o, peor aún, como algo inesencial. Esta forma de identidad puede ejemplificarse como sigue:

Decir que un planeta es un planeta, que el magnetismo es el magnetismo, que el espíritu es el espíritu, etc., lo considera cualquiera, con toda razón, como una manera de hablar idiota (Ibíd.).

Este fuerte calificativo, utilizado por Hegel tanto en la Enciclopedia como en la Ciencia de la lógica, obedece a que dicho razonar identificador no añade nada a la cosa más de lo que esta sea. Por ello, no es posible pensar que la filosofía sea una labor identificadora, pues en esta las determinaciones de la cosa o el sujeto están por fuera cuando de definirlos se trata. No obstante, y casi de manera obvia, Hegel no se contenta con mostrar esta falencia de la identidad del entendimiento, o identidad formal, es decir, no se conforma con mostrar la estructura de la Identidad, sino que da un paso más hacia la configuración de una identidad que será esencial y/o especulativa.

\section{Identidad y diferencia}

Un segundo momento del análisis de la Identidad, o de las determinaciones de la esencia, es el surgimiento de la diferencia como resultado de la refutación de la identidad abstracta. Para hablar de la diferencia hay que decir que esta es, en un primer momento, igual a la identidad, es decir, es abstracta en sí misma. Es una diferencia referida a sí misma y, por tanto, excluyendo de sí lo otro, "es diferencia de sí". Lo anterior significa que la diferencia, en un primer momento, también es un mero concepto vacío, pues al no referirse más que a sí misma no concibe la existencia de lo otro, tal cual sucedía con la identidad. Seguidamente, cuando Hegel se pregunta qué es lo diferente de la diferencia, es decir, qué es lo otro de ella, concluye obviamente que la identidad, por lo cual se deduce que dentro de esta está la diferencia, al igual que sucedió con la identidad. En este orden de ideas, es en la diferencia, como momento distinto de la identidad que sale de sí, se reconoce como diferente con respecto a sí y a lo diferente, donde están contenidos identidad y diferencia, de lo cual se concluye que "ambas juntas [identidad y diferencia] constituyen la diferencia" (Hegel, 1968. p. 367). Por ello, Hegel asegura que "la diferencia tiene los dos momentos: la identidad y la diferencia”. Pero, ¿por qué no asegurar lo mismo con respecto a la identidad? La respuesta para ello está en el hecho de que la identidad es aún, en un primer momento, una simple autorreferencialidad que sólo está cabe sí y que, por tal motivo, es un mero solipsismo. Cosa distinta su- 
cede con la diferencia, la cual implica, (hasta estructuralmente en el texto) una "conciencia" de la existencia de la diferencia y de la abstracción que implica el pensamiento identificador.

Igualmente, la diferencia entraña la inclusión de lo distinto en la formulación de un principio, así, por ejemplo, aparezcan de forma aislada $(\mathrm{A}=\mathrm{B})$. En este orden de ideas, en la proposición $\mathrm{A}=\mathrm{B}$ habría que decir que $\mathrm{A}$ es $\mathrm{B}$. Por tal motivo, y en sentido platónico, Hegel asegura que aquello que es común a los dos, a A y a B, es ser diferentes uno de otro, pero iguales a sí mismos, pues aún ninguno de los dos ha traspasado al otro. En consecuencia, es la diferencia la que contiene en sí a la identidad y a la diferencia o, dicho de otra forma, es la diferencia el género que traspasa la relación entre identidad y diferencia, concluyendo que lo idéntico es diferente de lo diferente y viceversa. Pero, como se ve, en la diferencia, en un primer momento, identidad y diferencia aparecen como opuestos independientes, es decir, diversos, y he aquí la primera forma en la cual aparece la diferencia. Así, es pertinente dar una mirada, así sea breve, a las distintas formas o momentos de decirse de la diferencia:

\subsubsection{Diversidad}

Son diversos los momentos de la diferencia, sin embargo, independientes y reflejados en sí mismos. Una relación diversa entre identidad y diferencia, por tanto, no implicará una reciprocidad entre los dos, sino una simple existencia del uno sin tomar en cuenta, ni siquiera, la existencia del otro. Así, lo idéntico es él, y lo diferente es él, lo cual presupone la inexistencia de una posible comunicación entre los mismos y, por lo tanto, las desmesuras y los excesos propios de su abstracción serán ilimitados e infinitos mientras esa apariencia de independencia permanezca en su fuero interno. No obstante, más allá de la mera enunciación de la diversidad, Hegel tematiza lo que él llama "principio de diversidad" que, opuesto obviamente al de la identidad, el cual reza que todas las cosas son iguales a sí mismas, asegura que "todo es diverso" o "no hay dos cosas que sean completamente iguales entre sí” (Hegel, 2000. § 117). Como es de notar, surge en la discusión sobre la diferencia un nuevo elemento, a saber, la igualdad. La razón de este surgimiento es que, en un momento más primario, más exterior, la diferencia no se presenta, o es puesta por la reflexión (extrínseca), como el otro de la identidad y/o como esencial a ésta, sino simplemente bajo la forma de la relación entre igualdad (Gleichheit) y desigualdad (Ungleichheit). Estas son determinaciones que no son en sí y por sí, es decir, son características propias de una relación inmediata de aquello que se da en la diversidad. Así, por ejemplo, lo igual y lo desigual no tienen ninguna relación posible entre sí, de tal suerte que lo igual es igual y lo desigual, por ende, desigual. En este orden de ideas, es necesario que, como consecuencia de esta 
estructura, surja un tercero (la reflexión, por ejemplo, dirá Hegel con respecto a Kant (Vide KrV A262/B318) que compare y diga qué es igual y qué es desigual, con base, por ejemplo, en expresiones como "en tanto", "por un lado", "respecto", etc. Así, de lo que se trata es que este tercero diga: estas dos cosas son iguales entre sí en tanto son diferentes de esta o estas otras. Por ello, algo es igual solo en tanto no sea desigual y viceversa. Por tanto, cada una de estas determinaciones de la diversidad, al ser extrínsecas, son autorreferenciales y meras mismidades vacías. No obstante, como se desprende de lo dicho hasta este momento sobre la igualdad y la desigualdad, el principio de la diversidad encuentra su nulidad en el momento mismo de la enunciación de su opuesto, es decir, cuando se afirma lo igual se hace gracias a distinguirlo de lo desigual, y viceversa. Por tal motivo, nada es absolutamente igual, pues es también desigual con respecto a otra cosa. Lo anterior, en el micro contexto de la diversidad, es lo mismo que puede concluirse de lo dicho por Hegel en relación con la identidad y la diferencia, sólo que allí se daba dicha conclusión de manera inmediata y, aquí, dicha inmediatez se determina y desarrolla.

\subsubsection{Oposición}

Un segundo momento que resulta luego del surgimiento y nulidad de la diversidad es la oposición, en la cual, en íntima relación con lo dicho inmediatamente antes, cada uno de los opuestos, identidad y diferencia, igualdad y desigualdad, "aparece en lo otro y sólo es en tanto lo otro también es" (Hegel, 2000. § 119). En este orden de ideas, en el ámbito de la oposición los opuestos ya no son independientes exclusivamente, es decir, ya no son en y por sí mismos en apariencia, sino que son el uno en el otro. No obstante, si bien son uno como conteniendo a lo otro, también se excluyen recíprocamente, pues son sólo momentos de la diferencia que, si bien son uno en virtud del otro, se excluyen en la medida en que la individualidad de cada uno de ellos aún no ha sido superada en virtud de que siguen siendo momentos, lo idéntico y lo diferente y, por ello, su independencia aún no está superada del todo, pues sólo se reconoce la presencia y necesidad del otro.

\subsubsection{La contradicción}

Por lo anterior, Hegel introduce, en contra de la tradición formal, la contradicción (Widerspruch) como eje fundamental de su filosofía. Esta (la contradicción) es necesaria y fundamental para la filosofía, la historia, el arte, etc., pues en ella, la relación de opuestos, que aparecen en la oposición como algo independiente, si bien su relación mutua y su reconocimiento de lo otro constituye un estadio superior con respecto a la mera diversidad, se soluciona (auflö- 
sung) con miras a que los opuestos sean tomados no como lados o momentos, sino como un todo que se constituye con base en el tránsito del uno al otro y a su relación con el todo. Dicho de otra forma, la inclusión de la contradicción en el discurso de Hegel tiene sentido y utilidad en la medida en que gracias a ella Identidad y diferencia no son independientes la una de la otra y, por lo tanto, una es en la medida en que esté dada la otra.

Por tal motivo, "la contradicción solucionada es así el fundamento" (Hegel, 1968. p. 382), en el cual la identidad ya no sólo es formal, en el sentido de ser resultado de la abstracción, sino que también se comprende a sí misma como negatividad, esto es, como diferencia, lo cual ya está contenido en la identidad formal de la esencia consigo misma, pero como algo que no se ha desplegado o, vale decir, es una relación de la cual aún la esencia no ha devenido consciente, para utilizar el contexto de la Fenomenología del espíritu. Por ello, "la identidad de la esencia consigo misma no es estática, sino que ella contiene previamente la negatividad en sí" o, lo que es lo mismo, "la esencia es un acto" (Cobben, 2006. p. 270), es decir, un constante automovimiento (Selbstbewegung) hacia sí misma.

De lo anterior se desprende, inevitablemente, que la relación entre identidad y diferencia o, lo que al comienzo de este escrito y en la llamada "gran lógica" Hegel nombró como "identidad de identidad y diferencia", que podría pensarse, en primera instancia, como una relación excluyente y, si se quiere, autoritaria de la identidad frente a su diferencia, es necesario pensarla, desde la propuesta lógica hegeliana, como una relación donde ninguno de los opuestos prima o se empodera sobre el otro, so pena de ser considerado como una auto posición producto de un entendimiento (Verstand) abstrayente, y por tanto, como una propuesta para pensar el fundamento (Grund), lo que fundamenta, como conteniendo en sí mismo, como unidad reconciliada, la identidad y la diferencia (Cfr. Cobben, 2006. pp. 249-250). Por ende, la formulación lógica de la identidad en Hegel no es formal sino real (Wirklichkeit), en el sentido de que dicha identidad es producto, y no mero presupuesto, de una relación recíproca con su otro, esto es, con la diferencia. Por ello, el problema de la identidad, su formulación lógica en la filosofía hegeliana, comporta una intención de Hegel por mostrar la identidad como algo real, en el sentido de que "contiene aquellas determinaciones anteriores y su diferencia, es decir, la intención de Hegel es concebir a la identidad en la diferencia y a ésta en aquella" (Hegel, 2000. § 118 z.) (especulativa) y no una mera identidad formal donde se excluye la diferencia.

\section{Conclusiones}

En principio, al menos dos conclusiones pueden extraerse de lo dicho: 
1.La noción hegeliana de la identidad no es un concepto que Hegel construye sin ningún tipo de conexión con la vida $\mathrm{y}$, por tanto, no es producto de una epifanía o alguna intención mística de su filosofía. Antes bien, la identidad busca resolver desde el ámbito de lo teórico un problema que tanto para Hegel como para varios de los más relevantes intelectuales de su época es de orden vital: la construcción de algo llamado Alemania, una nación en sentido estricto y no una mera ilusión.

2.A la luz de lo anterior, y para responder a la pregunta que da título a esta presentación, es imposible concluir, con Maker, que Hegel es un filósofo de la diferencia, pues si el elemento tomado para refutar la crítica a Hegel de ser el filósofo consumado de la identidad, y todo lo que ello implica, fue mostrar el lugar esencial que ocupa la diferencia, según Hegel, no sólo en la filosofía sino en la vida misma (los individuos no son mónadas, sino que gracias a lo que los diferencia pueden ser lo que cada uno es: identidad) y, por tanto, suprimir la diferencia sería suprimir eo ipso la identidad, otorgarle una presunta preponderancia a la diferencia sería negar ese momento positivo de la razón hegeliana, a saber, aquel en el cual se da la reconciliación de los opuestos.

\section{Bibliografía}

ADORNO, T. "Dialéctica negativa. La Jerga de la autenticidad”. Madrid: Akal, 2008. ASHTON, P. "The Beginning Before the Beginning: Hegel and the Activation of Philosophy". In: The Spirit of the Age: Hegel and the Fate of Thinking. Eds. Paul Ashton, Toula Nicolacopoulos and George Vassilacopoulos. Melbourne: re-press, 2008.

AVINERI, S. "Hegel's Theory of the Modern State". Cambridge: Cambridge University Press, 1972.

BRADY, T. "German Histories in the Age of Reformations, 1400-1650". New York: Cambridge University Press, 2009.

BRANDOM, R. "Some Hegelian Ideas of Note for Contemporary Analytic Philosophy". Hegel Bulletin, 35/1, 1-15 (2014). The Hegel Society of Great Britain, [Online]. Disponible en: http://bjsw.oxfordjournals.org/ (consultado el 25 septiembre de 2016) (doi:10.1017/hgl.2014.1).

COBBEN, P. "Hegel-Lexikon". Darmstadt: Wissenschaftliche Buchgesellschaft, 2006. DELEUZE, G. "Différence et Répétition". Paris: Presses Universitaires de France, 1993. DESMOND. W. "Beyond Hegel and Dialectic. Speculation, Cult and Comedy". New York: State University of New York Press, 1992.

HARRIS, H.S. "Hegel's Development. Toward the Sunlight 1770-1801". New York: Oxford University Press, 1972.

HEGEL, G. W. F. "Ciencia de la lógica”. Buenos Aires: Solar/Hachette, 1968.

HEGEL, G. W. F. "Diferencia entre los sistemas de filosofía de Fichte y Schelling". Madrid: Tecnos, 1990.

HEGEL, G. W. F. "Enciclopedia de las ciencias filosóficas en compendio para uso de sus clases". Madrid: Alianza Editorial, 2000.

HEGEL, G. W. F. "Lecciones sobre la filosofía de la historia universal. Tomo II. Madrid: Ediciones Altaya, 1994.

HEGEL, G. W. F. "Outlines of the Philosophy of Right". Oxford: Oxford University Press, 2008.

HEGEL, G. W. F. “Wissenschaft der Logik. Erster Teil. Die objektive Logik. Erstes 
Buch”. Frankfurt: Suhrkamp Verlag, 1969.

JAESCHKE, W. "Hegel-Handbuch. Leben, Werk, Schule". Stuttgart-Weimar: J. B. Metzler, 2003.

KANT, I. “Crítica de la razón pura”. Madrid: Taurus, 2005.

LONGUENSSE, B. "Hegel's Critique of Metaphysics". Cambridge: Cambridge University Press, 207.

MAKER, W. "Identity, Difference, and the Logic of Otherness". In: Identity and Difference. Studies in Hegel's Logic, Philosophy of Spirit, and Politics. Ed. Philip T. Grier. New York: State University of New York Press, 2007, pp. 15-30.

McDOWELLl, J. "Mind and World". Cambridge, MA: Harvard University Press, 1996. NOVALIS. "Philosophical Writings". New York: State University of New York Press, 1997.

NUZZO, A. "Dialectical Reason and Necessary Conflict: Understanding and the Nature of Terror". In: The Spirit of the Age: Hegel and the Fate of Thinking. Eds. Paul Ashton, Toula Nicolacopoulos and George Vassilacopoulos. Melbourne: re-press, 2008, pp. 21-38.

NUZZO, A. "Vagueness and Meaning Variance in Hegel's Logic". In: Hegel and the Analytic Tradition. Ed. Angélica Nuzzo. New York: Continuum International Publishing Group, 2010, pp. 61-82.

REDDING, P. "Analytic Philosophy and the Return of Hegelian Thought". Cambridge: Cambridge University Press, 2007.

ROECK, B. "Civic Culture and Everyday Life in Early Modern Germany". Netherlands: Brill, 2006.

SCHELLING, F. W. J. "Panorama general de la literatura filosófica más reciente". Ed. Vicente Serrano. Madrid: Abada Editores, 2006.

SCHILLER, F. (1975). "On the Aesthetic Education of Man". New York: Dover Publications, 2004.

SIEP, L. "Der Weg der Phänomenologie des Geistes. Ein einführender Kommentar zu Hegels 'Differenzschrift' und 'Phänomenologie des Geistes'”. Frankfurt: Suhrkamp Verlag, 2000.

SOLOMON, R. "In the Spirit of Hegel. A Study of G. W. F. Hegel's Phenomenology of Spirit”. New York: Oxford University Press, 1983.

STEWART, J. (ed.). "The Hegel Myths and Legends". Illinos: Northwestern University Press, 1996.

TAYLOR, A. J. P. "The Course of German History. A Survey of the Development of German History since 1815”. New York: Routledge, 2001.

TOEWS, J. E. "Hegelianism. The Path Toward Dialectical Humanism, 1805-1841". Cambridge: Cambridge University Press, 1980.

VON KLEIST, H. (1810). "Michael Kohlhaas”. In: Selected Prose of Heinrich von Kleist. New York: Archipielago Books, 2010. 